\title{
Nonintegrability and Chaos in the Anisotropic Manev Problem
}

\author{
Florin Diacu $^{1}$ and Manuele Santoprete ${ }^{2}$ \\ Department of Mathematics and Statistics \\ University of Victoria, P.O. Box 3045 \\ Victoria B.C., Canada, V8W 3P4
}

November 12, 2018

1e-mail: diacu@math.uvic.ca, phone: (250)721-6330, fax: (250)721-8962

2e-mail: msantopr@math.uvic.ca 


\begin{abstract}
The anisotropic Manev problem, which lies at the intersection of classical, quantum, and relativity physics, describes the motion of two point masses in an anisotropic space under the influence of a Newtonian force-law with a relativistic correction term. Using an extension of the Poincaré-Melnikov method, we first prove that for weak anisotropy, chaos shows up on the zero-energy manifold. Then we put into the evidence a class of isolated periodic orbits and show that the system is nonintegrable. Finally, using the geodesic deviation approach, we prove the existence of a large non-chaotic set of uniformly bounded and collisionless solutions.
\end{abstract}

PACS(2000): 05.45.Ac, 45.10.Hj, 45.50.Jf

Key words: Manev problem, anisotropy, chaos, geodesic deviation 


\section{Introduction}

The Manev problem studies the dynamics of two point masses in a flat space for an attraction law given by a potential of the form $\frac{A}{r}+\frac{B}{r^{2}}$, where $r$ is the distance between particles and $A, B>0$ are constants. The correction term to the Newtonian potential provides a classical approximation to general relativity [回], [2]. The anisotropic Manev problem, proposed by the first author in the mid 1990s [3], replaces the flat space with an anisotropic one.

The main reason for considering this question was that of seeking similarities between classical mechanics, quantum theory, and general relativity. Its study was inspired by the anisotropic Kepler problem introduced by Gutzwiller in the early 1970s [4], [5]. Gutzwiller aimed to find connections between classical and quantum mechanics. His interest in the anisotropic Kepler problem was aroused by an old quantum mechanical question related to a paper of Einstein [6].

Even though for a special class of (integrable) systems the Born-Sommerfeld-Einstein conditions [6] seemed appropriate for describing a Coulomb limit of quantum theory, it was unclear how to find a classical approximation for ergodic systems. Two qualities made the anisotropic Kepler problem suitable for addressing this issue: its chaotic character and its suitability to model various physical phenomena, as for example those encountered in certain semiconductors 四. Using a classical evaluation of the quantum Green's formula, Gutzwiller [7] found an approximate spectrum, in good agreement with previous quantum calculations. All these properties of the anisotropic Kepler problem raised hopes for the study of the Manev analogue, which also brought general relativity into the game.

Some of the qualitative features of the anisotropic Manev problem problem have already been studied. In a previous paper we proved the existence of an open, connected invariant manifold of uniformly bounded, collisionless orbits that lie within the negative-energy manifold [8]. In [3] it was shown that the differential equations describing the anisotropic Manev problem exhibit properties characteristic to all three aforementioned branches of 
physics. That paper also brought arguments favoring the nonintegrability of the system by putting into the evidence a network of heteroclinic orbits within the zero-energy manifold. Up to now, however, the existence of chaos and the nonintegrability of the system have not been rigorously proved.

The first result of this paper shows the presence of chaos for a small set of solutions within the zero-energy manifold (see Section III). We will base our proof on an extension of the Poincaré-Melnikov method developed in an earlier paper [9]. This method was used there to show the presence of chaos in several problems, including the restricted circular 3-body problem and the Gyldén problem. The latter had been earlier identified as a degenerate case for applying the Poincaré-Melnikov method [10]. In the 3-body problem the perturbation function, $W=W(r, \theta, t)$, where $(r, \theta)$ are the polar coordinates, appeared in its most general form. In the Gyldén problem this function was isotropic, $W=W(r, t)$. From the methodological point of view, we will now complete the applicative picture by offering a time-independent but anisotropic perturbation function of the form $W=W(r, \theta)$, which is specific to the anisotropic Manev problem (see Section II).

It is interesting to remark that different perturbations break up different symmetries, which affect the Melnikov integrals. In the general case both the homogeneity of time and the rotational symmetry are destroyed. This leaves us with two independent Melnikov conditions. In the Gyldén problem the rotational symmetry is preserved (the angular momentum is constant), which leads us to one Melnikov condition. In the present case only the time homogeneity is preserved (through the conservation of the Hamiltonian), so the only object of study is the other Melnikov condition (see Section III).

Our second result proves that the anisotropic Manev problem has no other analytic integrals beyond the Hamiltonian function. To show this, we use a method designed by Poincaré [11], [12], which we present in Section IV. The idea is to prove the existence of at least one isolated periodic orbit, which obstructs the occurrence of other analytic integrals. To apply Poincaré's method we need to write the perturbation as a Fourier series. This leads to some technical difficulties, which we overcome in Section V. In Section VI we prove the existence of many isolated periodic orbits in the hypothesis of weak anisotropy. We then show that this implies nonintegrability. The existence of periodic orbits, however, is an interesting result in itself, which raises hopes for applying Gutzwiller's formula to this problem in the context of semiclassical studies of the atom. 
In the last part of the paper we are concerned with the qualitative behavior of collisionless orbits for which the two particles stay close to each other. In Section VII we present the geodesic deviation method [13], which provides a criterion for excluding the presence of chaos. The idea is to transform the equations of motion into some equivalent ones. The new equations put the solutions of the given system in a one-to-one correspondence with the geodesics of a certain Riemannian manifold whose metric is determined by the initial equations of motion. Measuring the deviation of the geodesics, we can draw conclusions about the behavior of the initial solutions. If the geodesics diverge, chaos may appear; if they converge, chaos is ruled out. In Section VIII we show that there exists a positive-measure invariant manifold of orbits that fail to encounter collisions but for which the particles stay close to each other. Using the geodesic deviation method we then prove that the corresponding geodesics converge, thus showing that the orbits of this manifold are not chaotic.

\section{Equations of motion}

The (planar) anisotropic Manev problem is described by the Hamiltonian

$$
\mathcal{H}(\mathbf{q}, \mathbf{p})=\frac{1}{2} \mathbf{p}^{2}-\frac{1}{\sqrt{\mu q_{1}^{2}+q_{2}^{2}}}-\frac{b}{\mu q_{1}^{2}+q_{2}^{2}},
$$

where $b>0$ and $\mu>1$ are constants, $\mathbf{q}=\left(q_{1}, q_{2}\right)$ is the position of one body with respect to the other considered fixed at the origin of the coordinate system, and $\mathbf{p}=\left(p_{1}, p_{2}\right)=\dot{\mathbf{q}}$ is the momentum of the moving particle. The constant $\mu$ measures the strength of the anisotropy; the larger $\mu$, the higher the difference between the weakest and the strongest directions of attraction. For $\mu=1$ we recover the classical (isotropic) Manev problem of which we have a complete qualitative understanding [14], [1]. Therefore, if $\mu$ is only slightly larger than 1 , we are dealing with a weakly anisotropic case. Unlike in the isotropic problem, the angular momentum $K(t)=\mathbf{p}(t) \times \mathbf{q}(t)$ of the anisotropic case is not a first integral of the system. This is because the rotational invariance of the Hamiltonian breaks for $\mu>1$. Therefore we expect to encounter richer dynamics in the anisotropic case.

In the first part of this paper we will be interested in aspects related to weak anisotropy, i.e., in values of $\mu>1$ that are close to 1 . To put into 
the evidence the perturbative character of the anisotropy with respect to the isotropic problem, we introduce the notations $\epsilon=\mu-1$ and $r=\sqrt{q_{1}^{2}+q_{2}^{2}}$. For $\mu>1$ close to 1 , the quantity $\epsilon>0$ is small, so the Hamiltonian (1) can be viewed as describing a perturbation of the Manev problem by writing

$$
\mathcal{H}(\mathbf{q}, \mathbf{p})=\frac{1}{2} \mathbf{p}^{2}-\frac{1}{r}-\frac{b}{r^{2}}+\epsilon\left(\frac{1}{2 r}+\frac{b}{r^{2}}\right) \cos ^{2} \theta \equiv \mathcal{H}_{0}+\epsilon \mathcal{W}(r, \theta) .
$$

As in [10], we now consider the parabolic solutions of the unperturbed problem (defined by the Hamiltonian $\mathcal{H}_{0}$ ) that lie on the zero-energy manifold. These orbits, which play the role of homoclinic solutions corresponding to the critical point at infinity $(r=\infty, \dot{r}=0)$, satisfy the equations

$$
\dot{r}= \pm \frac{\sqrt{2 r-\left(k^{2}-2 b\right)}}{r}, \quad \dot{\theta}=\frac{k}{r^{2}}
$$

where $k \neq 0$ is the constant angular momentum of the unperturbed problem; the negative (positive) sign corresponds to $t<0(t>0)$. From (3) we get that

$$
\left\{\begin{array}{l} 
\pm t=\frac{k^{2}-2 b+r}{3} \sqrt{2 r-\left(k^{2}-2 b\right)}+\text { constant } \\
\theta= \pm 2 \arctan \frac{\sqrt{2 r-\left(k^{2}-2 b\right)}}{\sqrt{k^{2}-2 b}}+\text { constant }
\end{array}\right.
$$

Let

$$
R=R(t) \quad \text { and } \quad \Theta=\Theta(t)
$$

be the expressions of $r$ and $\theta$ with respect to $t$, obtained by inverting equations (4) and assuming that $R(0)=r_{\min }=k^{2} / 2$ and $\Theta(0)=\pi$. We will not need their expressions; as in [9], we only retain the information that $R$ is even and that $\Theta$ is odd.

\section{The Melnikov integral}

We now consider the problem defined by the Hamiltonian $\mathcal{H}$ given in (2). We will call homoclinic manifold the set of solutions of the unperturbed system that are doubly asymptotic to the point $r=\infty, \dot{r}=0$. For $k \neq 0$, the two-dimensional homoclinic manifold is described by $r=R\left(t-t_{0}\right)$ and $\vartheta=\Theta\left(t-t_{0}\right)+\theta_{0}$, with arbitrary constants $t_{0}$ and $\theta_{0}$. 
It is interesting to remark that in this problem we are dealing with negatively and positively asymptotic sets to the critical point $r=\infty, \dot{r}=0$ and that this point would correspond to the "critical point at infinity," obtained with the help of McGehee's transformation [15]

$$
r=\frac{1}{x^{2}}, \quad \dot{r}=y, \quad d t=\frac{1}{x^{3}} d s .
$$

But instead of using McGehee's technique, we will apply here the method introduced in [9]. It was shown there that under natural assumptions imposed on the perturbation function $\mathcal{W}(r, \theta)$, one can guarantee the existence of smooth solutions approaching the point $r=\infty, \dot{r}=0$ for $t \rightarrow \pm \infty$. It was also proved that the Poincaré map leads to infinitely many intersections of the stable and unstable manifold for the corresponding fixed point.

In our case the perturbation arising from a weak anisotropy vanishes as $r \rightarrow \infty$. This happens because $\mathcal{W}(r, \theta) \sim 1 / r$, which is exactly condition (18) in [9]. So we can write the Melnikov condition as in [9], with the only amendment of dropping the time dependence. Therefore the two Melnikov integrals become

$$
M_{1}\left(\theta_{0}\right)=\int_{-\infty}^{+\infty}\left[\dot{R}(t) \frac{\partial \mathcal{W}\left(R(t), \Theta(t)+\theta_{0}\right)}{\partial r}+\dot{\Theta}(t) \frac{\partial \mathcal{W}\left(R(t), \Theta(t)+\theta_{0}\right)}{\partial \theta}\right] \mathrm{d} t=0
$$

and

$$
M_{2}\left(\theta_{0}\right)=\int_{-\infty}^{+\infty} \frac{\partial \mathcal{W}\left(R(t), \Theta(t)+\theta_{0}\right)}{\partial \theta}=0 .
$$

Since $W$ vanishes as $t \rightarrow \pm \infty$, the first Melnikov condition takes the form

$$
M_{1}\left(\theta_{0}\right)=\int_{-\infty}^{+\infty} \frac{\partial \mathcal{W}\left(R(t), \Theta(t)+\theta_{0}\right)}{\partial t} \mathrm{~d} t \equiv 0
$$

$M_{1}$ is identically zero since the perturbation function $\mathcal{W}$ is independent of time. This simplifies our discussion because, unlike in the general case, we only need to find the zeroes of the integral in (8).

It is significant to remark, and easy to check, that the previous conditions can also be written in terms of the first integrals of the unperturbed problem as

$$
M_{1}\left(\theta_{0}\right)=\int_{-\infty}^{+\infty}\left\{\mathcal{H}_{0}, \mathcal{W}\right\}(\ldots) \mathrm{d} t=0
$$


and

$$
M_{2}\left(\theta_{0}\right)=\int_{-\infty}^{+\infty}\{K, \mathcal{W}\}(\ldots) \mathrm{d} t=0
$$

Notice that $M_{1}\left(\theta_{0}\right)$ is identically zero. This resembles a results obtained for the Gyldén problem [9], [10] and is related to the symmetries of the problem. In the Gyldén problem the perturbation is independent of the angle $\theta$ but depends on time. This means that the perturbation alters the homogeneity of time, so the Hamiltonian is not an integral of motion anymore, but it leaves the rotational invariance intact; thus the angular momentum is still conserved. Therefore there is only one condition here, given by relation (9). On the other hand in the anisotropic Manev problem the anisotropy of the space alters only the rotational symmetry but not the homogeneity of time. This also leads to only one condition, given by relation (8).

For the anisotropic Manev problem the Melnikov condition $M_{2}$ takes the form

$$
M_{2}\left(\theta_{0}\right)=\int_{-\infty}^{+\infty} \sin \left[2\left(\Theta(t)+\theta_{0}\right)\right]\left(\frac{1}{R(t)}+\frac{b}{R(t)^{2}}\right) d t=0
$$

Using some trigonometry the integral can be written as

$$
M_{2}\left(\theta_{0}\right)=I_{1} \cos 2 \theta_{0}+I_{2} \sin 2 \theta_{0},
$$

where $I_{1}$ and $I_{2}$ are defined by the relations

$$
\left\{\begin{array}{l}
I_{1}=\int_{-\infty}^{+\infty}\left(\frac{1}{R(t)}+\frac{b}{R(t)^{2}}\right) \sin 2 \Theta(t) d t \\
I_{2}=\int_{-\infty}^{+\infty}\left(\frac{1}{R(t)}+\frac{b}{R(t)^{2}}\right) \cos 2 \Theta(t) d t
\end{array}\right.
$$

Since $R$ and $\Theta$ are even and odd functions of time, respectively, the integrand of $I_{1}$ is an odd function. Therefore $I_{1} \equiv 0$, and finally $M_{2}$ can be rewritten as

$$
M_{2}\left(\theta_{0}\right)=I_{2} \sin 2 \theta_{0} .
$$

Since we found simple zeroes of the Melnikov function, the following result holds.

Theorem 1 In some invariant set contained in the zero-energy manifold, the weakly anisotropic Manev problem exhibits chaotic dynamics. 
This type of chaotic behavior, which usually takes place for a small set of orbits, is induced by the infinitely many intersections of the stable and unstable manifolds for the Poincaré map associated to the critical point at infinity. Let us notice that the Poincaré-Birkhoff-Smale theorem [16] does not directly apply to this situation, which is degenerate. However, the existence of Smale horseshoes and of positive topological entropy is not restricted to hyperbolic equilibria. This phenomenon is also encountered in nonhyperbolic cases, as for example those arising when dealing with area-preserving perturbations [17]. Thus, Theorem 1 adds to the class of results describing chaotic dynamics near degenerate equilibria.

\section{Poincaré's method}

In this section we will present a classical result of Poincaré [11], [12] in connection with the nonexistence of additional analytic integrals in Hamiltonian systems. Based on the investigation of long periodic solutions, this criterion is suitable for proving the nonintegrability of the anisotropic Manev problem for weak anisotropy, as we will see in Section $\mathrm{V}$.

First we need to write the unperturbed system, i.e. the (isotropic) Manev problem, in terms of action-angle variables [18]. The action variables are given by

$$
\left\{\begin{array}{l}
I=\frac{1}{2 \pi} \oint p_{r} d r=-\sqrt{K^{2}-2 b}+\frac{1}{2} \sqrt{\frac{2}{|h|}} \\
K=q_{1} p_{2}-q_{2} p_{1},
\end{array}\right.
$$

where $h$ is the energy constant and $K$ is the angular momentum. These variables are defined for $h<0$ and $K^{2}>2 b$. The associated angular frequencies are

$$
\left\{\begin{array}{l}
\omega_{I}=\frac{1}{\left(I+\sqrt{K^{2}-2 b}\right)^{3}} \\
\omega_{K}=\frac{K}{\sqrt{K^{2}-2 b}\left(I+\sqrt{K^{2}-2 b}\right)^{3}} .
\end{array}\right.
$$

With the help of the action-angle variables, the unperturbed Hamiltonian $\mathcal{H}_{0}$ defined by relation (2) can then be written as

$$
H_{0}(I, K)=-\frac{1}{2\left(I+\sqrt{K^{2}-2 b}\right)^{2}},
$$


so the unperturbed equations of motion take the form

$$
\left\{\begin{array}{l}
\dot{I}=\frac{\partial H_{0}}{\partial \phi}=0 \\
\dot{K}=\frac{\partial H_{0}}{\partial \theta}=0 \\
\dot{\phi}=-\frac{\partial H_{0}}{\partial I}=\omega_{I} \\
\dot{\theta}=-\frac{\partial H_{0}}{\partial K}=\omega_{K}
\end{array}\right.
$$

Recall that the eigenvalues $\lambda$ of the monodromy operator of a $T$-periodic solution are called characteristic multipliers and that the numbers $\alpha$ defined by $\lambda=\exp (\alpha T)$ are called characteristic exponents [16], [18]. We would like to show the nonexistence of other analytic integrals, independent of the Hamiltonian $H_{0}$. Our main theoretical tool in proving the nonintegrability of the perturbed system is the following result, proved in [11.

Theorem 2 Suppose that the two degree of freedom Hamiltonian system with Hamiltonian function $H$ has two first integrals $H$ and $F$ that are independent along a periodic solution. Then four characteristic exponents vanish.

One exponent vanishes because the system is autonomus. The second vanishes due to the presence of the first integral $H$. If the remaining exponents are different from zero, the periodic solution is called nondegenerate. It is well known that nondegenerate periodic orbits are isolated [12].

To establish the existence of a large number of isolated periodic orbits, suppose that for $I=I^{0}$ and $K=K^{0}$, the frequencies $\omega_{I}$ and $\omega_{K}$ of the unperturbed problem are commensurable and that $\omega_{I} \neq 0$. Then the perturbing function $W\left(I, K, \omega_{I} t, \omega_{K} t+\lambda\right)$, defined by $\mathcal{W}$ given in (2) and then transformed with the help of the action-angle variables, is periodic in $t$ and has period $T$. Consider its average

$$
\bar{W}\left(I^{0}, K^{0}, \lambda\right)=\frac{1}{T} \int_{0}^{T} W\left(I, K, \omega_{I} t, \omega_{K} t+\lambda\right) d t .
$$

Then the following theorem, also due to Poincaré [11], establishes the existence of isolated periodic orbits.

Theorem 3 Assume that the following conditions are satisfied:

(1) The Hamiltonian is nondegenerate at the point $I=I^{0}, K=K^{0}$,

(2) for some $\lambda=\lambda^{*}$ the derivative $\partial \bar{W} / \partial \lambda=0$, but $\partial^{2} \bar{W} / \partial \lambda^{2} \neq 0$. 
Then, for small $\epsilon \neq 0$, the perturbed Hamiltonian system has a T-periodic solution that depends analytically on the parameter $\epsilon$ and for $\epsilon=0$ coincides with the periodic solution

$$
I=I^{0}, \quad K=K^{0}, \quad \phi=\omega_{I} T, \quad \theta=\omega_{K} t+\lambda^{*}
$$

of the unperturbed system. The two charateristic exponents $\pm \alpha$ of this solution admit a convergent series expansion in power series of $\sqrt{\epsilon}$,

$$
\alpha=\alpha_{1} \sqrt{\epsilon}+\alpha_{2} \epsilon+\alpha_{3} \epsilon \sqrt{\epsilon}+\ldots
$$

where

$$
\omega_{I}^{2} \alpha_{1}^{2}=\frac{\partial^{2} \bar{W}\left(\lambda^{*}\right)}{\partial \lambda^{2}}\left(\omega_{I}^{2} \frac{\partial^{2} H_{0}}{\partial K^{2}}-2 \omega_{I} \omega_{K} \frac{\partial^{2} H_{0}}{\partial I \partial K}+\omega_{K}^{2} \frac{\partial^{2} H_{0}}{\partial I^{2}}\right) .
$$

problem. Using Theorem 2, one can show the dependence of the functions $H_{0}$ and $F_{0}$ on the set of unperturbed tori $I=I_{0}, K=K_{0}$ that satisfy the conditions of Theorem 3 and the relation

$$
\left(\omega_{I}^{2} \frac{\partial^{2} H_{0}}{\partial K^{2}}-2 \omega_{I} \omega_{K} \frac{\partial^{2} H_{0}}{\partial I \partial K}+\omega_{K}^{2} \frac{\partial^{2} H_{0}}{\partial I^{2}}\right) \neq 0 .
$$

So the following result holds.

Theorem 4 Under the hypotheses of Theorem 3 and relation (19), the Hamiltonian system (16) does not admit a first integral $F$ independent of $H$ that can be written as a formal power series of the form $\sum_{s \geq 0} F_{s}(I, K, \phi, \theta) \epsilon^{s}$ with analytic coefficients $F_{s}$.

\section{Fourier series of the perturbation}

In order to apply these results to our perturbed problem, we first need to write the perturbation function as a Fourier series. For this let us first notice that $r$ can be expressed in parametric form with respect to time. Following the same procedure used in [19] for the Kepler problem, we can write

$$
t=\sqrt{\frac{1}{2|h|}} \int \frac{r d r}{\sqrt{-r^{2}+\frac{r}{|h|}-\frac{K^{2}-2 b}{2|h|}}} .
$$


With the substitutions

$$
A=\frac{1}{2|h|} \quad \text { and } \quad A^{2} e^{2}=\left(\frac{1}{2|h|}\right)^{2}-\frac{K^{2}-2 b}{2|h|}
$$

the above relation takes the form

$$
t=\sqrt{A} \int \frac{r d r}{\sqrt{A^{2} e^{2}-(r-A)^{2}}} .
$$

With the change of variables $r-A=-A e \cos \eta$, the integral assumes the simpler expression

$$
t=A^{\frac{3}{2}} \int(1-e \cos \eta) d \eta=A^{\frac{3}{2}}(\eta-e \sin \eta)+\text { constant. }
$$

If we choose the time in such a way that the addition constant is zero, then

$$
r=A(1-e \cos \eta) \quad \text { and } \quad t=A^{\frac{3}{2}}(\eta-e \sin \eta) .
$$

We can now write the constants $A$ and $e$ in terms of the action variables as

$$
A=\frac{1}{2|h|}=\left(I+\sqrt{K^{2}-2 b}\right)^{2} \quad \text { and } \quad e^{2}=\frac{I\left(I+2 \sqrt{K^{2}-2 b}\right)}{\left(I+\sqrt{K^{2}-2 b}\right)^{2}} .
$$

Recall that the Fourier series expansion of a function $F$ has the form

$$
F(\eta)=\sum_{l=-\infty}^{+\infty} A_{l} e^{i l \eta}, \text { where } A_{l}=\frac{1}{2 \pi} \int_{0}^{2 \pi} F(\eta) e^{-i l \eta} d \eta
$$

and that that the Bessel function $J_{m}(z)$ is given by

$$
J_{m}(z)=\frac{1}{2 \pi} \int_{0}^{2 \pi} \cos (m u-z \sin u) d u=(-1)^{m} J_{-m}(z) .
$$

It is now easy to show [19] that

$$
\frac{A}{r}=1+2 \sum_{l=1}^{\infty} J_{l}(l e) \cos l \eta
$$

where $\eta=\omega_{I} t$. Therefore

$$
\frac{A^{2}}{r^{2}}=1+4 \sum_{l=1}^{\infty} J_{l}(l e) \cos l \eta+4 \sum_{l, m=1}^{\infty} J_{l}(l e) J_{m}(m e) \cos l \eta \cos m \eta
$$


Using some trigonometry we obtain that

$$
\begin{gathered}
\frac{A^{2}}{r^{2}}=1+4 \sum_{l=1}^{\infty} J_{l}(l e) \cos l \eta(l+m)+ \\
2 \sum_{l, m=1}^{\infty} J_{l}(l e) J_{m}(m e)[\cos \eta(l+m)+\cos \eta(l-m)] .
\end{gathered}
$$

More computations lead us to the relation

$$
\frac{A^{2}}{r^{2}}=1+\sum_{l=1}^{\infty}\left(J_{l}(l e)\right)^{2}+4 \sum_{l=1}^{\infty}\left(J_{l}(l e)+\frac{A_{l}}{2}+B_{l}\right) \cos \eta l-2 A_{1} \cos \eta
$$

where

$$
A_{l}=\sum_{\substack{\alpha, \beta \\ \alpha+\beta=l}} J_{\alpha}(\alpha e) J_{\beta}(\beta e) \quad \text { and } \quad B_{l}=\sum_{\substack{\alpha, \beta \\ \alpha-\beta=l}} J_{\alpha}(\alpha e) J_{\beta}(\beta e) .
$$

We can write the the perturbation function $W$ as a series of the form

$$
W\left(I, K, \omega_{I} t, \omega_{K} t\right)=\left(D+\sum_{l=1}^{\infty} C_{l} \cos l \omega_{I} t\right) \cos ^{2} \omega_{K} t
$$

where

$$
\begin{gathered}
C_{l}=\frac{4 b}{A^{2}}\left[J_{l}(l e)\left(\frac{A}{b}+1\right)+\frac{A_{l}}{2}+B_{l}\right] \text { for } l \neq 1, \\
C_{1}=\frac{4 b}{A^{2}}\left[J_{l}(l e)\left(\frac{A}{b}+1\right)+\frac{A_{l}}{2}+B_{l}\right]-2 A_{1},
\end{gathered}
$$

and

$$
D=\frac{b}{A^{2}}\left(1+\sum_{l=1}^{\infty}\left(J_{l}(l e)\right)^{2}\right)
$$

Using some trigonometry, the above relation becomes

$$
W\left(I, K, \omega_{I} t, \omega_{K} t\right)=D+\frac{1}{2} \sum_{\substack{l=0 \\ m= \pm 1,0}}^{\infty} C_{l, m} \cos \left(l \omega_{I}+2 m \omega_{K}\right) t
$$

where $C_{l, m}=C_{l}$ for all $l, m \neq 0, C_{0,1}=D$, and $C_{0,-1}=0$. 


\section{Periodic orbits and nonintegrability}

We can now apply the method of the previous section to the anisotropic Manev problem. As we are going to show, all the assumptions of Theorem 3 are satisfied, so for weak anisotropy the existence of isolated periodic orbits follows. This leads to the following result.

Theorem 5 For small $\epsilon \neq 0$, the weakly anisotropic Manev problem has a T-periodic solution that depends analytically on the parameter $\epsilon$. For $\epsilon=0$ this solution coincides with the periodic solution

$$
I=I^{0}, \quad K=K^{0}, \quad \phi=\omega_{I} T, \quad \theta=\omega_{K} t+\lambda^{*}
$$

of the unperturbed system, where $\lambda^{*}$ can take the values $0, \frac{\pi}{2}, \pi, \frac{3 \pi}{2}$. Moreover, the two charateristic exponents $\pm \alpha$ of the solution admit a convergent series expansion in $\sqrt{\epsilon}$, given by (17), where $\alpha_{1}$ is defined by (18).

Proof. First we will show that the assumptions of Theorem 3 are satisfied. It is easy to see that the unperturbed Hamiltonian is non-degenerate. Indeed,

$$
\operatorname{det}\left(\begin{array}{cc}
\frac{\partial^{2} H_{0}}{\partial I^{2}} & \frac{\partial^{2} H_{0}}{\partial I \partial K} \\
\frac{\partial^{2} H_{0}}{\partial K \partial I} & \frac{\partial^{2} H_{0}}{\partial K^{2}}
\end{array}\right)=\frac{b}{\left(K^{2}-2 b\right)^{3 / 2}\left(I+\sqrt{K^{2}-2 b}\right)^{3}} .
$$

We are left with computing the average $\bar{W}$ of the perturbation function $W$ for values of the action variables whose frequencies of the unperturbed problem are commensurable. Let $\omega_{K}=\frac{p}{q} \omega_{I}$. Then we can write the perturbation as

$$
D+\frac{1}{2} \sum_{\substack{l=0 \\ m= \pm 1,0}}^{\infty} C_{l, m} \cos \left[\left(l+2 m \frac{p}{q}\right) \omega_{I} t+2 m \lambda\right] .
$$

It is clear that the only $\lambda$-dependent terms that survive after averaging are the resonant ones, i.e., the terms with the property that $l+2 m \frac{p}{q}=0$. It is easy to see that we have to consider only the terms with $m= \pm 1$. This implies $q= \pm 1, \pm 2$ and then $l= \pm 2 p, \pm p$. These terms are of the form

$$
C_{ \pm 2 p, \pm 1} \cos (2 \lambda) \quad \text { and } \quad C_{ \pm p, \pm 1} \cos (2 \lambda),
$$

SO

$$
\frac{\partial \bar{W}}{\partial \lambda}=G \sin 2 \lambda \quad \text { and } \quad \frac{\partial^{2} \bar{W}}{\partial \lambda^{2}}=2 G \cos 2 \lambda,
$$


where

$$
G=2\left(C_{-2 p, 1}+C_{2 p, 1}+C_{-p, 1}+C_{p, 1}\right) .
$$

This means that for $\lambda^{*}=0, \frac{\pi}{2}, \pi, \frac{3 \pi}{2}$, the second condition of Theorem 3 is satisfied if $G$ is not identically zero.

We will now show that, in general, $G$ cannot be identically zero. Indeed, notice first that $G$ depends on $A$ and is analytic in $A$. But $G$ cannot be identically zero beyond a discrete set of values of $A$ unless the Bessel function is itself identically zero. However, the Bessel function has only a discrete set of zeroes. Therefore, generically, $G$ cannot be identically zero, so the existence of isolated periodic orbits follows. This completes the proof.

For the anisotropic Manev problem, we are now able to prove the nonexistence of first integrals that are independent of $H$ and analytic in the parameter $\epsilon$. In more formal terms, the following result holds.

Theorem 6 For small values of $\epsilon$, the weakly anisotropic Manev problem does not admit a formal first integral $F$ independent of $H$ that can be written as a power series $\sum_{s \geq 0} F_{s}(I, K, \phi, \theta) \epsilon^{s}$ whose coefficients $F_{s}$ are analytic functions.

Proof. According to Theorem 4, the only thing we need to prove is that inequality (19) is satisfied. Then using Theorem 5 the result follows. In our case inequality (19) can be written as

$$
\omega_{I}^{3}-2 \omega_{K}^{2} \omega_{I}+\frac{\omega_{K}^{4}}{\omega_{I}}+b \frac{\omega_{I}^{11 / 3}}{\omega_{K}} \neq 0 .
$$

If we substitute $\omega_{K}=\frac{p}{q} \omega_{I}$ (where $\omega_{I} \neq 0$ and $p \neq 0$ ) we obtain that (19) is always verified except for the solutions of the equation

$$
\omega_{I}^{3}\left(\frac{p}{q}\right)^{5}-2 \omega_{I}^{3}\left(\frac{p}{q}\right)^{3}+\omega_{I}^{3}\left(\frac{p}{q}\right)+b \omega_{I}^{8 / 3}=0 .
$$

But this equation has at most five distinct solutions, which do not affect the outcome since, if eliminating them, the remainig set is still dense in the real line. This completes the proof. 


\section{Geodesic deviation}

In this and the following section we will show that if the particles do not collide but stay close enough together, the motion is not chaotic. This is somewhat surprising since one expects that, as in celestial mechanics or atomic physics, chaos appears because of near-collision approaches. Of course, our result does not exclude the possibility that chaos is determined by only brief passages close to collision.

Our proof is based on a local criterion due to Szczȩsny and Dobrowolski [13]. The idea is to rewrite the equations of motion in terms of the geodesic equation with respect to the Jacobi metric and to measure the local tendency of the geodesics to diverge or converge. If they diverge, chaos may appear, but if they converge, chaos is impossible.

Let us first outline the ideas that lead to the above mentioned criterion. In general, for a Hamiltonian function

$$
\mathcal{H}(\mathbf{q}, \mathbf{p})=\frac{1}{2} g^{i j}(\mathbf{q}) \mathbf{p}_{i} \mathbf{p}_{j}+U(\mathbf{q})
$$

where $g^{i j}$ are the components of the covariant tensor corresponding to the Riemannian metric $g_{i j} d \mathbf{q}^{i} \otimes \mathbf{q}^{j}$, with $\mathbf{p}_{i}=g_{i j} \mathbf{q}^{i}$, the equations of motion can be written as the Euler-Lagrange system

$$
\ddot{\mathbf{q}}^{i}+\Gamma_{k l}^{i} \dot{\mathbf{q}}^{k} \dot{\mathbf{q}}^{l}=-g^{i k} \partial_{k} U(\mathbf{q})
$$

in which $\Gamma_{k l}^{i}$ is the Levi-Civita connection for the metric $g_{i j}$. The energy relation $\mathcal{H}(\mathbf{q}, \mathbf{p})=h$ foliates the phase space in codimension-one manifolds. Let us fix an $h$ and define an open set $\mathbf{D}_{h}$ of the configuration space as $\mathbf{D}_{h}=\{\mathbf{q} \mid U(\mathbf{q})<h\}$. It can be shown that the orbits of (20) are orthogonal to the boundary of $\mathbf{D}_{h}$. Let us define the Jacobi metric $\hat{g}^{i j}=2(h+U) g^{i j}$ and the parameter $s$ such $\frac{d s}{d t}=2(h+U)$. It can be shown that on the set $\mathbf{D}_{h}$, equations (20) are equivalent to the geodesic equations

$$
\frac{d^{2} \mathbf{q}^{i}}{d s^{2}}+\hat{\Gamma}^{i}{ }_{j k} \frac{d \mathbf{q}^{j}}{d s} \frac{d \mathbf{q}^{k}}{d s}=0
$$

where $\hat{\Gamma}^{i}{ }_{j k}$ is the Christoffel symbol with respect to the metric $\hat{g}^{i j}$. Note that $\hat{g}_{i j}(\mathbf{q}(s)) \frac{d \mathbf{q}^{i}}{d s} \frac{d \mathbf{q}^{j}}{d s}=1$. Equations (21) can be further written as

$$
\hat{\nabla}_{\mathbf{u}} \mathbf{u}=\mathbf{0}
$$


where $\mathbf{u}$ is the tangent vector to the geodesic and $\hat{\nabla}$ is the covariant derivative with respect to the Jacobi metric. Aiming to understand the relative motion of geodesics in a domain inside $\mathbf{D}_{h}$, we take a curve transversal to the geodesics, then take a copy of it by transporting each point of the initial curve along the geodesic, and measure in this way the local relative deviation of the geodesics. It can be proved that, in classical form, the measure of the deviation is given by the solution of the Jacobi equation [20]

$$
\frac{d^{2} x}{d s^{2}}=-\mathcal{K}(s) x
$$

where $x$ is the variable that measures the deviation and $\mathcal{K}$ is the Gauss curvature at $x(s)$. If $\mathcal{K}>0$ the geodesics approach each other, whereas if $\mathcal{K}<0$ they diverge. For 2-degree of freedom systems the curvature is given by the formula [13]

$$
\mathcal{K}=\frac{g^{i j}\left(\partial_{i} V\right)\left(\partial_{j} V\right)+(h-U) \Delta U}{4(h-U)^{3}},
$$

where $\Delta=\partial_{1}^{2}+\partial_{2}^{2}$ is the Laplacian operator. In [13] the following result is proved.

Theorem 7 If $\mathcal{K}$ given by formula (23) is negative, the geodesics diverge and chaos may appear; if $\mathcal{K}$ is positive, the geodesics converge and chaos cannot take place.

We will further use this result to investigate the possibility of encountering chaotic motion in the neighborhood of collisions.

\section{Absence of chaos near collision}

In order to apply Theorem 1 to the equations of motion of the anisotropic Manev problem, let us first show that the hypothesis that leads to the geodesic deviation equation (22) is satisfied. For this we need to make sure that the geodesics do not reach the boundary of the set $\mathbf{D}_{h}$, in other words we must isolate a set of solutions with this property. This will be achieved while proving the following result, which shows that collisionless solutions that keep the particles close together cannot be chaotic. 
Theorem 8 There exists a positive-measure set of orbits that are free of collisions and for which the distance between particles does not exceed a certain uniform bound. Within this set, chaotic motion is ruled out.

Proof. In the case of the anisotropic Manev problem, the expression of the kinetic energy shows that the metric is flat and that $g_{i j}=1$ for $i=j$ and $g_{i j}=0$ for $i \neq j$. Therefore the curvature $\mathcal{K}$ given by formula (23) takes the form

$$
\mathcal{K}=\frac{|\nabla U|^{2}+(h-U) \Delta U}{4(h-U)^{3}},
$$

where $U(\mathbf{q})=-\frac{1}{\sqrt{\mu q_{1}^{2}+q_{2}^{2}}}-\frac{b}{\mu q_{1}^{2}+q_{2}^{2}}$. Let us now see that there exists a set of solutions, whose projection in the configuration space lies inside $\mathbf{D}_{h}$. These will form an open set of uniformly bounded and collisionless solutions.

For this consider the analytic diffeomorphic transformation of the dependent variables,

$$
\left\{\begin{array}{l}
r=\left(q_{1}^{2}+q_{2}^{2}\right)^{\frac{1}{2}} \\
\varphi=\arctan \left(q_{2} / q_{1}\right) \\
v=\dot{r}=q_{1} p_{1}+q_{2} p_{2} \\
u=r^{2} \dot{\varphi}=q_{1} p_{2}-q_{2} p_{1},
\end{array}\right.
$$

and the analytic diffeomorphic transformation of the independent variable

$$
d \tau=r^{-1} d t .
$$

Notice that $r$ and $\varphi$ are polar coordinates, whereas $v$ and $u$ represent the rescaled radial and tangential components of the velocity. In these new variables, the equations of motion take the form

$$
\left\{\begin{array}{l}
r^{\prime}=v \\
v^{\prime}=2 h r+\left(\mu \cos ^{2} \varphi+\sin ^{2} \varphi\right)^{-1 / 2} \\
\varphi^{\prime}=r^{-1} u \\
u^{\prime}=\frac{\mu-1}{2} r^{-1}\left[\left(\mu \cos ^{2} \varphi+\sin ^{2} \varphi\right)^{-3 / 2}+2 b\left(\mu \cos ^{2} \varphi+\sin ^{2} \varphi\right)^{-2}\right] \sin 2 \varphi
\end{array}\right.
$$

and the energy relation $\mathcal{H}(\mathbf{q}, \mathbf{p})=h$ becomes

$$
u^{2}+v^{2}-2 r\left(\mu \cos ^{2} \varphi+\sin ^{2} \varphi\right)^{-1 / 2}-2 b\left(\mu \cos ^{2} \varphi+\sin ^{2} \varphi\right)^{-1}=2 h r^{2} .
$$

The new dependent variables $(r, v, \varphi, u) \in(0, \infty) \times \mathbb{R} \times S^{1} \times \mathbb{R}$ are functions of the fictitious time $\tau$, so the prime denotes from now on differentiation with respect to this new independent variable. 
Let us first notice that there exist two constants, $m_{1}$ and $m_{2}$ with $0<$ $m_{1}<m_{2}<\infty$, such that along any solution $(r, v, \varphi, u)$ of system $(26)$, the function of $\tau$ given by $\mu \cos ^{2} \varphi+\sin ^{2} \varphi$ satisfies the relations

$$
m_{1}<\mu \cos ^{2} \varphi+\sin ^{2} \varphi<m_{2},
$$

for all $\tau$ for which $\varphi$ is defined. Consequently there exist two constants, $M_{1}$ and $M_{2}$, with $0<M_{1}<M_{2}<\infty$, such that

$$
M_{1}<\left(\mu \cos ^{2} \varphi+\sin ^{2} \varphi\right)^{-1 / 2}<M_{2}
$$

for all $\tau$. From the first two equations of system (26), we obtain the nonhomogeneous second-order equation

$$
r^{\prime \prime}-2 h r=\left(\mu \cos ^{2} \varphi+\sin ^{2} \varphi\right)^{-1 / 2},
$$

which represents a forced harmonic oscillator. We will further ignore the last two equations of system (26) and retain only the information that the forcing function $\left(\mu \cos ^{2} \varphi+\sin ^{2} \varphi\right)^{-1 / 2}$ is uniformly bounded from above and away from zero from below, as relations (28) indicate.

We will further restrict our analysis to the invariant manifold of solutions of negative energy, $h<0$, which obviously exists according to relation (27). Solving the homogeneous equation $r^{\prime \prime}-2 r h=0$ and then applying to equation (29) the method of variation of parameters, we obtain the general solution of equation (29) in the form

$$
\begin{aligned}
r(\tau) & =\left(c_{1}+\frac{1}{\sqrt{-2 h}} \int_{0}^{\tau} \frac{\cos (\sqrt{-2 h} \sigma)}{\sqrt{\mu \cos ^{2} \varphi(\sigma)+\sin ^{2} \varphi(\sigma)}} d \sigma\right) \sin (\sqrt{-2 h} \tau) \\
& +\left(c_{2}-\frac{1}{\sqrt{-2 h}} \int_{0}^{\tau} \frac{\sin (\sqrt{-2 h} \sigma)}{\sqrt{\mu \cos ^{2} \varphi(\sigma)+\sin ^{2} \varphi(\sigma)}} d \sigma\right) \cos (\sqrt{-2 h} \tau) .
\end{aligned}
$$

If, for every solution of constants $c_{1}$ and $c_{2}$, we apply to each of the above integrals the intermediate-value theorem and then use some trigonometry we obtain

$$
\begin{gathered}
r(\tau)=\left(c_{1}^{2}+c_{2}^{2}\right)^{\frac{1}{2}} \cos \left(\omega \tau-\omega_{0}\right)- \\
\frac{\sin ^{2}(\sqrt{-2 h} \tau)}{2 h \sqrt{\mu \cos ^{2} \varphi\left(\tau_{1}\right)+\sin ^{2} \varphi\left(t a u_{1}\right)}}-\frac{\cos ^{2}(\sqrt{-2 h} \tau)}{2 h \sqrt{\mu \cos ^{2} \varphi\left(\tau_{2}\right)+\sin ^{2} \varphi\left(\tau_{2}\right)}},
\end{gathered}
$$

where $\tau_{1}$ and $\tau_{2}$ belong to the interval $(0, \tau), \omega=\sqrt{-2 h}$ and $\omega_{0}=\arctan \frac{c_{2}}{c_{1}}$. Relations (28) and (30) allow us to draw the conclusion that for any solution $(r, v, \varphi, u)$ of system $(26)$, the inequalities

$$
\sqrt{c_{1}^{2}+c_{2}^{2}} \cos \left(\omega \tau-\omega_{0}\right)-\frac{M_{1}}{2 h}<r(\tau)<\sqrt{c_{1}^{2}+c_{2}^{2}} \cos \left(\omega \tau-\omega_{0}\right)-\frac{M_{2}}{2 h}
$$


take place for all $\tau$ for which the solution is defined.

Let us now fix some $h_{0}<0$, as close to 0 as we like, and define the set $\Lambda\left(h_{0}\right)$ of solutions formed by the union of all sets $\Lambda_{h}$, for $h$ in the interval $\left(-\infty, h_{0}\right)$, where each set $\Lambda_{h}$ contains all the solutions given by (30) that satisfy the inequality

$$
c_{1}^{2}+c_{2}^{2}<\frac{M_{1}^{2}}{4 h^{2}}
$$

Obviously, this is an open, nonempty, and connected invariant manifold of system (26). From (31) it follows that, for every solution of this manifold, $r$ is positive and bounded, therefore the orbits are collisionless and bounded. Let us denote by $\bar{\Lambda}\left(h_{0}\right)$ the projection of $\Lambda\left(h_{0}\right)$ into the configuration space. For $\mu$ fixed, suitable choices of $c_{1}, c_{2}$, and $h$ provide solutions with $q_{1}$ and $q_{2}$ small enough such that $U(\mathbf{q})<h_{0}$. Moreover these choices can be made such that $r$ is as close to 0 as we like but still positive. Obviously, the set of the corresponding solutions has positive measure. Let us denote by $\Gamma\left(h_{0}\right)$ the corresponding projection of the set of such solutions in the configuration space. Obviously $\Gamma\left(h_{0}\right)$ is contained in $\bar{\Lambda}\left(h_{0}\right)$ as well as in $\mathbf{D}_{h_{0}}$. This shows that the hypothesis leading to the criterion of the previous section is satisfied. A straightforward computation yields

$$
\mathcal{K}=-\frac{3(h-U)\left(\mu^{2} q_{1}^{2}+q_{2}^{2}\right)}{\left(\mu q_{1}^{2}+q_{2}^{2}\right)^{5 / 2}}-\frac{[1-8 b(h-U)]\left(\mu^{2} q_{1}^{2}+q_{2}^{2}\right)}{\left(\mu q_{1}^{2}+q_{2}^{2}\right)^{3}}+\frac{4 b^{2}\left(\mu^{2} q_{1}^{2}+q_{2}^{2}\right)}{\left(\mu q_{1}^{2}+q_{2}^{2}\right)^{4}}+\frac{4 b\left(\mu^{2} q_{1}^{2}+q_{2}^{2}\right)}{\left(\mu q_{1}^{2}+q_{2}^{2}\right)^{7}} .
$$

The first term is always negative and the second can also be negative, depending on the fixed value of $b$. But since the last two terms are positive and the powers at their denominators exceed those of the denominators of the first two terms, for sufficiently small $q_{1}$ and $q_{2}$, the curvature $\mathcal{K}$ is positive. We can always define $\Gamma\left(h_{0}\right)$ such that the configurations corresponding to the solutions with corresponding $q_{1}$ and $q_{2}$ are contained in $\Gamma\left(h_{0}\right)$. Therefore, according to Theorem 7, the set of collisionless solutions for which the bodies stay close enough together, does not exhibit chaotic behavior. This completes the proof.

Acknowledgements. Florin Diacu was supported in part by the Pacific Institute for the Mathematical Sciences and by the NSERC Grant OGP0122045. Manuele Santoprete was sponsored by a University of Victoria Fellowship. 


\section{References}

[1] F. Diacu, V. Mioc, and C. Stoica, Nonlin. Analysis 41, 1029-1055 (2000).

[2] W. Thirring, A Course in Mathematical Physics I, Classical Dynamical Systems (Springer Verlag, New York 1978). A. Lacomba, J. Libre and A. Nunes, systems", in The Geometry of Hamiltonian Systems, Proceedings of a Workshop held June 5-16, T. Raţiu, ed., (Springer Verlag, New York 1991). Y. Hagihara, Celestial Mechanics (MIT Press, Cambridge, Ma, 1975), Vol II, Part I.

[3] S. Craig, F. Diacu, E.A. Lacomba, and E. Perez, J. Math. Phys. 40, 1359-1375 (1999).

[4] M.C. Gutzwiller, J. Math. Phys. 12, 343-358 (1971), 14, 139-152 (1973), 18, 806-823 (1977).

[5] M.C. Gutzwiller, Chaos in Classical and Quantum Mechanics (Springer Verlag, New York, 1990).

[6] A. Einstein, Verhandl. Deutsch. Phys. Gesellsch. 19, 82-92 (1917).

[7] M.C. Gutzwiller, Physica D 5, 183-207 (1982).

[8] F. Diacu, J. Phys. A (submitted).

[9] G. Cicogna and M. Santoprete, J. Math. Phys. 41, 805-815 (2000).

[10] F. Diacu and D. Şelaru, problem" J. Math. Phys. 39, 6537-6546 (1998).

[11] H. Poincaré, New Methods of Celestial Mechanics, (Amer. Inst. Phys., 1993), vol. I, Ch. 5.

[12] V.I. Arnold, ed., Dynamical System III, Encyclopedia of Mathematical Sciences, vol. 3 (Springer, New York, 1988).

[13] J. Szczȩsny and T. Dobrowolski, approach to chaos, Ann. Phys. 277, 161-176 (1999).

[14] J. Delgado, F. Diacu, E.A. Lacomba, A. Mingarelli, V. Mioc, E. Perez, and C. Stoica, J. Math. Phys. 37, 2748-2760 (1996). 
[15] R. McGehee, J. Diff. Eqns. 14, 70-88 (1973).

[16] J. Guckenheimer and P. Holmes, Nonlinear Oscillations, Dynamical Systems and Bifurcations of Vector Fields (Springer Verlag, New York, 1983). F. Diacu and P. Holmes, Celestial Encounters - The Origins of Chaos and Stability (Princeton University Press, Princeton, NJ, 1996).

[17] K. Burns and H. Weiss, Comm. Math. Phys. 172, 95-118 (1995).

[18] S. Wiggins, Global Bifurcations and Chaos (Springer Verlag, New York, 1989).

[19] A. Wintner, The Analytical Foundations of Celestial Mechanics (Princeton University Press, Princeton NJ, 1941). L.D. Landau and E.M. Lifshitz, Mechanics (Pergamon, Oxford, 1969).

[20] V.I. Arnold, Mathematical Methods of Classical Mechanics (Springer Verlag, New York, 1989). 